\title{
THE EFFECTS OF INTRANASAL OXYTOCIN ON
}

\section{THE NEURAL CIRCUITRY OF SOCIAL COGNITION}

\section{Diana Moura ${ }^{1,2}$, Ana ISABel Bessa ${ }^{2}$, Joaquim Cerejeira ${ }^{3}$}

${ }^{1}$ Psychiatry Department, Coimbra Hospital University Centre, Portugal; ${ }^{2}$ Faculty of Medicine, University of Coimbra, Portugal;

${ }^{3}$ Coimbra Institute for Clinical and Biomedical Research

\section{INTRODUCTION}

Oxytocin (OT) is a neuropeptide synthesized in the hypothalamus, known for its multiple functions in the human body. OT plays an important part in social bonding and sexual reproduction and, in recent years, emerging evidence suggests that OT modulates neuronal activity in brain regions that play a main role in social cognitive processes. This effect appears to be due to the binding of OT to specific receptors in particular brain regions that have not been fully explored. Advances in human non-invasive neuroimaging techniques have allowed researchers to begin to reveal the neurobiological basis of human social behavior. The identification of the brain regions affected by OT during a social task might begin to shed a light on the neurobiological foundations of its effects in human social cognition and behavior.

\section{OBJECTIVE}

The present study aims to assess and summarize the current knowledge on how exogenous OT influences the activity of different brain areas during social cognitive tasks.

\section{METHODS}

\section{Search Strategy}

We conducted a systematic review assessing placebocontrolled studies that used functional magnetic resonance imaging to investigate the effects of intranasal OT in the brain of healthy subjects. we used a systematic search strategy following the PRISMA guidelines for systematic reviews. we identified nineteen original studies that met our eligibility criteria.

\section{Data Analysis}

The selected studies were screened with the purpose of determining OT's effect on the activity of different brain areas. Collected information: sample size, population features (including mean age, gender and race), study design, dose of OT, time between OXT administration and $\mathrm{fMRI}$ scan, total scan duration, description of the social task and the brain areas which responded to the administration of OXT.

\section{Selection criteria}

Inclusion criteria: 1) primary research studies using intranasal OT in humans and measuring brain activity with $\mathrm{fMRI}$ during social-cognitive tasks based on visualstimuli; 2) double-blind, placebo-controlled design; 3) studies with healthy participants with no history of neurological, psychiatric or endocrine disease, no intake of medication that could interfere with the experiment and no drug or alcohol abuse.

\section{Assess the risk of bias}

To assess the methodological quality of the included studies, the authors determined the risk of bias using the Cochrane Collaboration's tool. We found the risk of bias was reasonably small for each individual study.

\section{CONCLUSION}

\section{RESULTS}

The aforementioned results refer to the specific neural effects of exogenous OT compared to placebo. Table 1 summarizes the main brain areas affected by OT.

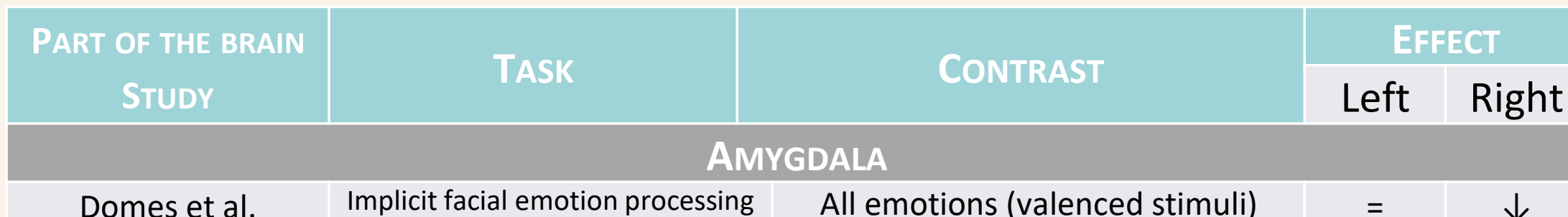

Domes et al. Implicit facial emotion processing All emotions (valenced stimuli)

$\downarrow$

Domes et al.

Kirsch et al.

Petrovic et al.

\begin{tabular}{|l|l|}
\multicolumn{2}{c}{ processing } \\
\hline Domes et al. & Explicit facial emotion processing
\end{tabular}

Fearful>Neutral

Fearful>Neutra

Direct gaze

$\uparrow$

$\downarrow$

$\downarrow$

Domes et al.

Luo et al.

Rilling et al.

Implicit facial processing

Cooperation game/interaction \section{CII}

Empathy for pain Implicit facial processing Cooperation game/interaction

\section{HIPPOCAMPUS}

Kanat et al.

Rilling et al.

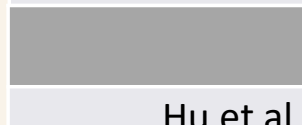

Hu et al.

Reinforcement association learning task

Wittfoth-Schardt et al. Implicit facial processing CAUdATE Nucleus

Fearful>Neutral

Happy>Neutral

Females: $\mathrm{FN}>\mathrm{NN}$ Reciprocated cooperation

$\begin{array}{ll}\uparrow & = \\ \uparrow & = \\ \uparrow & = \\ \uparrow & \uparrow\end{array}$

Feng et al.

Cooperation game/interaction

Reciprocated cooperation (males>females)

\begin{tabular}{|l|l|l|l|l|}
\hline & \multicolumn{4}{c}{ (males>females) } \\
\hline Rilling et al. & Cooperation game/interaction & Reciprocated cooperation & $d$ & $\uparrow$ \\
\hline Rilling et al. & Cooperation game/interaction & Reciprocated cooperation & $=$ & $\uparrow$ \\
\hline
\end{tabular}

(males $>$ females)

\begin{tabular}{|c|c|c|c|c|}
\hline \multicolumn{5}{|c|}{ TEMPORAL LOBE } \\
\hline Groppe et al. & Social incentive delay task & Social reward antecipation & $\uparrow$ & $=$ \\
\hline Hecht et al. & Animations of moving shapes & Social relationships>Physical relationship & $\downarrow$ & $=$ \\
\hline Petrovic et al. & $\begin{array}{l}\text { Aversely conditioned face } \\
\text { processing }\end{array}$ & Direct gaze & $=$ & $\downarrow$ \\
\hline \multicolumn{5}{|c|}{ FRONTAL LOBE } \\
\hline Groppe et al. & Social incentive delay task & Social punishment anticipation & $\downarrow$ & $\downarrow$ \\
\hline \multirow[t]{2}{*}{ Hu et al. } & Altruistic interaction & Help>Punish (computer decision) & $\uparrow$ & $=$ \\
\hline & & Help>Punish (self-decision) & $\downarrow$ & $\downarrow$ \\
\hline decreased ac & ncreased activity; =: no a & rations in the activity. & & \\
\hline
\end{tabular}

In recent years, several OT-based fMRI studies have revealed promising findings, allowing a significant advance to our knowledge of the brain regions in which social cognition is modulated by OT. In this review, we found evidence of OT's effects in social brain areas in every included study. The most frequently identified brain region whose activity was affected by OT was the amygdala, followed by the frontal and temporal lobes. Altogether, our findings allow the development of an approach that could be used to demonstrate differential OT modulation of brain circuitry between psychiatric patients and healthy controls, regarding the identification of treatment targets and OT-based therapies. We rely on future work to consider these factors in order to unify the current findings and to define the modulatory role of OT on the neurobiological basis of human sociality.

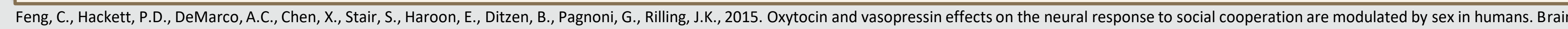
Imaging Behav. 9, 754-764.

Gamer, M., Zurowski, B., Buchel, C., 2010. Different amygdala subregions mediate valence-related and attentional effects of oxytocin in humans. Proc. Natl. Acad. Sci. 107, 9400-9405 Hu, Y., Scheele, D., Becker, B., Voos, G., David, B., Hurlemann, R., Weber, B., 2016. The Effect of Oxytocin on Third-Party Altruistic Decisions in Unfair Situations: An fMRI Study. Sci. Rep. 6 Kanat, M., Heinrichs, M., Mader, I., Van Elst, L.T., Domes, G., 2015. Oxytocin Modulates Amygdala Reactivity to Masked Fearful Eyes. Neuropsychopharmacology 40, $2632-2638$.

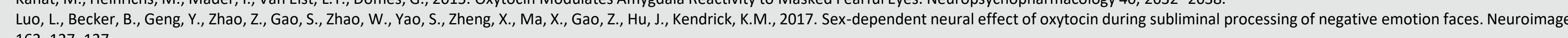
$162,127-137$

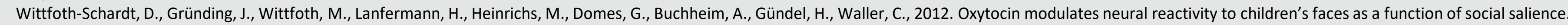
Neuropsychopharmacology 37, 1799-1807 